Pacific Journal of Mathematics

ON THE MULTIPLICATIVE EXTENSION PROPERTY

ard Albert Cleveland and Sandra Cleveland 


\title{
ON THE MULTIPLICATIVE EXTENSION PROPERTY
}

\section{Richard and Sandra Cleveland}

\begin{abstract}
A subspace $M$ of a Banach algebra $B$ is said to have the multiplicative extension property (abbr. m.e.p.) if whenever $L$ is a linear functional on $M$ of norm not greater than one, $L$ is the restriction to $M$ of a multiplicative linear functional on $B$. This property is considered in two settings-the measure algebra $M(G)$ of a suitable group, and the disc algebra $A(D)$ of functions analytic in the unit disc with continuous boundary values. The following theorems are proved.

Theorem 2. If $Q$ is a compact subset of $G$ such that $M_{c}(Q)$ has the m.e.p., then (i) for every nonzero $t \in G$, the set $Q \cap(Q-t)$ has $\mu$-measure zero for every continuous measure $\mu$ on $G$, and (ii) $m(Q)=0$, where $m$ is the Haar measure for $G$.

Theorem 3. Suppose $G$ contains an independent Cantor set. Then there exists a compact subset $Q$ of $G$ such that for infinitely many $t \neq 0, Q \cap(Q-t)$ is countably infinite, and $M_{c}(Q)$ has the m.e.p.

Theorem 4. There exist infinite dimensional subspaces of $A(D)$ with the m.e.p.

These last two theorems are proved by constructing examples using a special decomposition of the Cantor set. This decomposition is presented in a separate section to simplify notation.
\end{abstract}

The multiplicative extension property was formulated by Hewitt in [1] after Hewitt and Kakutani [2] had given examples of such subspaces of the measure algebra of certain locally compact groups. In [1] Hewitt poses the problem of characterizing the subspaces with the m.e.p. in a general Banach algebra, and points out that the question was open even for the algebra $C(X)$ of all continuous complex valued functions on a compact Hausdorff space $X$. Later Phelps [4], who calls the m.e.p. "property $(H)$ ", announced such a characterization for $C(X)$. Phelps has shown that a closed subspace $A$ of $C(X)$ has the m.e.p. if and only if $X$ is homeomorphic to a symmetric compact convex subset of a locally convex space and $A$ is the space of linear functions on $X$. If $B$ is any commutative Banach algebra with unit and maximal ideal space $X$, and if $M$ is a closed subspace of $B$ with the m.e.p., then the Gelfand transform is an isometric isomorphism of $M$ onto a closed subspace of $C(X)$ which has the m.e.p. Thus Phelps' result characterizes arbitrary subspaces with the m.e.p., in a sense. However, this result gives no information on whether a given algebra has subspaces

Received March 8, 1965. 
with the m.e.p., or how to find such subspaces if they do exist.

The purpose of this paper is to give some further examples of subspaces with the m.e.p. We consider two algebras - the measure algebra of a suitable group $G$, and the dise algebra $A$ of all functions analytic in the unit dise with continuous boundary values. In $M(G)$ we consider the following question: what conditions must be satisfied by a compact set $Q$ in order that the space $M_{c}(Q)$ of all continuous measures supported on $Q$ have the m.e.p.? This question seems to be very difficult, and we cannot give a complete answer, but we do arrive at some necessary conditions on $Q$. Roughly speaking, such a set $Q$ must be fairly independent from a measure theoretic point of view, while an example is constructed to show that it can be rather dependent from an algebraic point of view. This is done in Section 3. In Section 4 we consider the disc algebra $A$ where we construct an infinite dimensional subspace $M$ with the multiplicative extension property. This subspace $M$ has the interesting property that if $f$ and $g$ are linearly independent elements of norm 1 in $M$, then $g$ assumes every value in some closed disc on the set of zeroes of $f$.

While the two examples of this paper are very different in character, they are both constructed by means of a special decomposition of the Cantor set into an uncountable family of disjoint perfect subsets. While this decomposition is not new, it is presented in Section 2 in order to provide a common notation for the later constructions.

2. Decomposition of the Cantor Set. Let $C$ be the Cantor ternary set. If we let $x(n)$ denote the $n$th ternary digit of $x \in C$, where $x(n)=0$ or $x(n)=2$, then each $x$ has a unique ternary expansion. For each $x \in C$, let

$$
E_{x}=\{y \in C: y(2 n)=x(n) \text { for all } n \in N\} .
$$

The following statements are easily verified:

(1) For each $x \in C, E_{x}$ is a nonempty perfect subset of $C$.

(2) If $x \neq y$ then $E_{x} \cap E_{y}=\varnothing$, and $C=\bigcup_{x \in o} E_{x}$.

(3) If $t_{n} \in E_{x_{n}}(n=1,2, \cdots$,$) , and t_{n} \rightarrow t_{0}$ where $t_{0} \in E_{x_{0}}$, then $x_{n} \rightarrow x_{0}$.

For each $x \in C$ we define a map $h_{x}$ from $E_{0}$ to $E_{x}$ as follows: for $y \in E_{0}$ and $k \in N$,

$$
\left[h_{x}(y)\right](k)= \begin{cases}x(k / 2), & \text { if } k \text { is even } \\ y(k), & \text { if } k \text { is odd. }\end{cases}
$$

Then it is easy to check that each $h_{x}$ is a homeomorphism of $E_{0}$ onto $E_{x}$, and $h_{0}$ is the identity map. Moreover, we have

(4) For $x \in C, y \in E_{0}$ let $H(x, y)=h_{x}(y)$. Then $H$ is continuous 
from $C \times E_{0}$ onto $C$.

Indeed, if $\left\{x_{n}: n \in N\right\} \subset C,\left\{y_{n}: n \in N\right\} \subset E_{0}, x_{n} \rightarrow x_{0}$, and $y_{n} \rightarrow y_{0}$, and $k \in N$, then we may choose $\nu$ so that for $n \geqq \nu, y_{n}(j)=y_{0}(j)$ for $j \leqq 2 k+1$, while $x_{n}(j)=x_{0}(j)$ for $j \leqq k$. Thus, for $j \leqq 2 k+1$ we have $H\left(x_{n}, y_{n}\right)(j)=H\left(x_{0}, y_{0}\right)(j), n \geqq \nu$. Since $k$ is arbitrary, we see that

$$
\lim H\left(x_{n}, y_{n}\right)=H\left(x_{0}, y_{0}\right)
$$

3. Subspaces of $M(G)$. In this section we consider the algebra $M(G)$ of measures on a nondiscrete locally compact abelian group $G$. We are interested in what properties a compact $Q \subset G$ must have in order that $M_{c}(Q)$, the subspace of continuous measures carried on $Q$, have the m.e.p. Hewitt and Kakutani show in [2] that if $P$ is an independent Cantor set in $G$ then $M_{c}(P \cup(-P))$ has the m.e.p. The proof of this relies heavily on the independence of $P$. Our results show that in general if $M_{c}(Q)$ has the m.e.p., then $Q$ must be "almost independent" in a sense to be made more precise. Then we give an example that shows that the set $Q$ can be, at the same time, rather dependent.

THeOREM 1 (Hewitt and Kakutani). Suppose $Q$ is a compact subset of $G$ such that $M_{c}(Q)$ has the m.e.p. Then if $k, l$ are distinct positive integers and $\lambda_{1}, \cdots, \lambda_{k}$ and $\mu_{1}, \cdots, \mu_{l}$ are nonnegative measures in $M_{c}(Q)$ and if $\pi, \rho$ are arbitrary invertible elements of norm 1 in $M(G)$, then the measures $\lambda=\lambda_{1} * \cdots * \lambda_{k} * \pi$ and $\mu=\mu_{1} * \cdots * \mu_{l} * \rho$ are mutually singular.

Hewitt and Kakutani prove this in [2] (Theorem 4.8) in the case that $Q=P \cup(-P)$. But their proof only uses the fact that $M_{c}(Q)$ has the m.e.p. and goes over to the general case without change.

THeOREM 2. If $Q$ is a compact subset of $G$ such that $M_{c}(Q)$ has the m.e.p., then

(i) for every nonzero $t \in G$, the set $Q \cap(Q-t)$ has $\mu$-measure zero for every continuous measure $\mu$ on $G$.

(ii) $m(Q)=0$, where $m$ is the Haar measure for $G$.

Proof. (ii) is a simple consequence of (i) since $m(Q \cap(Q-x))$ is continuous as a function of $x$. To see (i), suppose on the contrary that $\mu(Q \cap(Q-t)) \neq 0$ for some $t \neq 0$ and $\mu \in M_{c}(G)$. Let $\nu$ be the restriction of $\mu$ to subsets of $Q \cap(Q-t)$. Then both $\nu$ and $\nu_{t}=\nu * \varepsilon_{t}$ are in $M_{c}(Q)$. Now $\nu$ and $\nu_{t}$ cannot be linearly independent, since then there would be a multiplicative linear functional $h$ on $M(G)$ such that 
$h(\nu)=0, h\left(\nu_{t}\right) \neq 0$, by the m.e.p. But $h\left(\nu_{t}\right)=h(\nu) h\left(\varepsilon_{t}\right)$. Hence $\nu_{t}=\alpha \cdot \nu$ for some $\alpha \neq 0$. Now let $V$ be a symmetric neighborhood of 0 such that $t \notin V+V$. Then choose $x \in Q \cap(Q-t)$ so that $\nu(x+V) \neq 0$. Let $\lambda$ be the restriction of $\nu$ to subsets of $U=x+V$. Since $U \cap(U+t)=\varnothing$ and since $\lambda_{t}$ is carried on $U+t$, we see that $\lambda_{t} \neq \alpha \cdot \lambda$, for any $\alpha$. But $\lambda$ and $\lambda_{t}$ are both in $M_{c}(Q)$, and by the same argument as above with $\lambda$ in place of $\nu, \lambda_{t}=\alpha \cdot \lambda$, for some $\alpha$. This contradiction establishes the result.

In particular, we see from (i) that if $Q$ is metrizable then $Q \cap(Q-t)$ must be at most countable for every $t \neq 0$. This is the sense of "almost independence" mentioned above.

We give an example to show that $Q \cap(Q-t)$ can actually be countably infinite infinitely often. For this example we suppose $G$ contains an independent set $P$ homeomorphic to Cantor's set. This is always true, for example, if $G$ contains arbitrarily small elements of infinite order [5, p. 100].

THEOREM 3. Suppose $G$ contains an independent Cantor set. Then there exists a compact subset $Q$ of $G$ such that for (countably) infinitely many $t \neq 0, Q \cap(Q-t)$ is countably infinite, and $M_{c}(Q)$ has the m.e.p.

Proof. Let $P$ be an independent Cantor set in $G$ and let $P=S \cup T$ where $S$ and $T$ are disjoint Cantor sets. Let $\sigma$ and $\tau$ be homeomorphisms of $C$ onto $S$ and $T$, respectively, and for $x \in C$, let $S_{x}=\sigma\left(E_{x}\right)$ and $T_{x}=\tau\left(E_{x}\right)$. Let $c_{0}<c_{1}<c_{2}<\cdots$ be a monotone sequence in $E_{0}$ and denote the limit by $c_{\infty}$. Choose a sequence $\left\{U_{n}: n=0,1,2, \cdots\right\}$ of disjoint clopen (relative to $E_{0}$ ) sets such that $c_{n} \in U_{n}$. Write $U_{\infty}=\left\{c_{\infty}\right\}$. For $0 \leqq n \leqq \infty, x \in C$, let

$$
\begin{aligned}
a_{n}(x) & =\sigma\left(h_{x}\left(c_{n}\right)\right) \\
b_{n}(x) & =\tau\left(h_{x}\left(c_{n}\right)\right) \\
B_{n}(x) & =\tau\left(h_{x}\left(U_{n}\right)\right) \quad \text { if } \quad n \neq 0 \\
B_{0}(x) & =\sigma\left(E_{x}\right)=\sigma\left(h_{x}\left(E_{0}\right)\right) . \\
A_{n}(x) & =B_{n}(x)-b_{n}(x)+a_{n}(x) .
\end{aligned}
$$

Let $K$ be any countable closed subset of $C$ and let

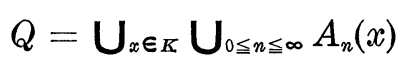

We break the rest of the proof into several steps.

Lemma 1. $Q$ is closed.

Proof. Indeed, consider the map $\gamma$ on $\bigcup\left\{U_{n}: 0 \leqq n \leqq \infty\right\}$ to 
$\left\{c_{n}: 0 \leqq n \leqq \infty\right\}$ defined by

$$
\gamma(t)=\sum_{0 \leqq n \leqq \infty} c_{n} 1_{U_{n}}(t),
$$

where $1_{A}$ denotes the characteristic function of $A$. Then $\gamma$ is continuous. By Remark (4) of Section 2, the mapping

$$
F(x, t)=\tau\left(h_{x}(t)\right)-\tau\left(h_{x}(\gamma(t))\right)+\sigma\left(h_{x}(\gamma(t))\right)
$$

is a continuous mapping of the compact set $K \times \bigcup\left\{U_{n}: 0 \leqq n \leqq \infty\right\}$ onto $Q$. Thus $Q$ is closed.

LEMMA 2. $m(Q)=0$.

Proof. Each element of $Q$ is of the form $p-b+a$, where $p, b$, $a$ are in $P$. Hence $Q$ is contained in the subgroup generated by $P$, and, since $P$ is independent, the Haar measure of this subgroup is zero. (C.f. [5], p. 108).

Lemma 3. $Q \cap(Q-t)$ is infinite for infinitely many $t \neq 0$.

Proof. For each $n \geqq 1$ and $x \in K$,

$$
\begin{aligned}
a_{n}(x) \in A_{n}(x) & \cap\left[A_{0}(x)+b_{0}(x)-a_{0}(x)\right] \\
\subset Q & \cap\left(Q-t_{x}\right)
\end{aligned}
$$

where $t_{x}=a_{0}(x)-b_{0}(x) \neq 0$.

We also note here that $Q \cap(Q-t)$ is at most countable for any $t \neq 0$.

Lemma 4. Suppose $\mu_{1}, \cdots, \mu_{r}$ are nonnegative continuous measures concentrated on disjoint subsets $D_{1}, \cdots, D_{r}$ of $Q$. Let $\lambda=\mu_{1}^{n 1} * \cdots * \mu_{r}^{n r}$ and $\nu=\mu_{1}^{m 1} * \cdots * \mu_{r}^{m r}$. Then $\lambda$ and $\mu$ are mutually singular unless $n_{i}=m_{i}$ for $1 \leqq i \leqq r$.

Proof. There are only a countable number of elements of $Q$ of the form

$$
\begin{array}{cc}
b_{n}(x)-b_{n}(x)+a_{n}(x), & n \geqq 1 \\
\text { or } \quad a_{k}(x)-b_{0}(x)+a_{0}(x), \quad k \geqq 0 .
\end{array}
$$

Since the $\mu_{i}$ are continuous, we may assume without loss of generality that no $D_{i}$ contains a point of this type. $\lambda$ is concentrated on $D_{\lambda}=$ $n_{1} D_{1}+\cdots+n_{r} D_{r}$ and $\nu$ is concentrated on $D_{\nu}=m_{1} D_{1}+\cdots+m_{r} D_{r}$. We shall show that $D_{\lambda} \cap D_{\nu}=\varnothing$ unless $n_{i}=m_{i}$ for $1 \leqq i \leqq r$.

Let $D=D_{\lambda} \cap D_{\nu}$. Each element of $D$ has two representations 


$$
\begin{aligned}
& \left(y_{1}^{1}+\cdots+y_{n_{1}}^{1}\right)+\cdots+\left(y_{1}^{r}+\cdots+y_{n_{r}}^{r}\right) \\
= & \left(z_{1}^{1}+\cdots+z_{m_{1}}^{1}\right)+\cdots+\left(z_{1}^{r}+\cdots+z_{m_{1}}^{r}\right),
\end{aligned}
$$

where $y_{k}^{i}$ and $z_{k}^{i}$ are in $D_{i}$. Each element of $D_{i}$ is of the form

$$
\beta_{n}(x)-b_{n}(x)+a_{n}(x)
$$

where $\beta_{n}(x) \in B_{n}(x)$, and $\beta_{n}(x) \neq b_{n}(x), n \geqq 1$, and $\beta_{0}(x) \neq a_{k}(x), k \geqq 0$. Thus (*) is an equation between two combinations of elements of $P$. Since $P$ is independent, the coefficients of distinct terms on either side must be equal. For fixed $n$ and $x$ suppose $a_{n}(x)$ appears $k$ times on the left hand side of $\left(^{*}\right)$, i.e., the coefficient of $a_{n}(x)$ on the left is $k$. This means that there are $k$ elements on the left of the form

$$
\beta_{n}^{i}(x)-b_{n}(x)+a_{n}(x)
$$

and $k$ elements on the right of the form

$$
\gamma_{n}^{i}(x)-b_{n}(x)+a_{n}(x)
$$

where $\beta_{n}^{i}(x)$ and $\gamma_{n}^{i}(x)$ are in $B_{n}(x)$, since $a_{n}(x)$ can occur only in this way. Since none of the $\beta^{\prime}$ s or $\gamma^{\prime}$ s can occur in any other way on either side of the equation, we must have

$$
\sum_{i=1}^{k} \beta_{n}^{i}(x)=\sum_{i=1}^{k} \gamma_{n}^{i}(x)
$$

and so the $\beta_{n}^{i}(x)$ are just a permutation of the $\gamma_{n}^{i}(x)$. Thus the $y_{j}^{i}$ that belong to a fixed $A_{n}(x)$ are just a permutation of the $z_{j}^{i}$ that belong to $A_{n}(x)$. It follows that the $y^{\prime}$ s are a permutation of the $z^{\prime} \mathbf{s}$ and, by the disjointedness of $D_{1}, \cdots, D_{r}, n_{i}=m_{i}$ for $1 \leqq i \leqq r$.

Lemma 5. $M_{c}(Q)$ has the m.e.p.

The rest of the proof is exactly the same as the proof of Theorem 5.4.1 in [5], so we will not duplicate that proof here.

By a slight modification of the proof of Theorem 6.2 in [2], one can show that if $P$ is, in addition, a Kronecker set, then the multiplicative extensions of linear functionals are in the closure of the dual of $G$.

It is natural to ask whether the above construction of the set $Q$ could be modified in such a way that $Q \cap(Q-t)$ is infinite for uncountably many $t$. The following example shows that $M_{c}(Q)$ may fail to have the m.e.p. even though $Q \cap(Q-t)$ has at most two elements for every $t \neq 0$.

Consider the set

$$
Q=\bigcup_{x \in o}\left[A_{0}(x) \cup A_{1}(x) \cup A_{2}(x)\right],
$$


where the notation is as above, except for $Q$. This $Q$ is a compact set of Haar measure zero, and $Q \cap(Q-t)$ has at most two points for $t \neq 0$, but $M_{c}(Q)$ does not have the m.e.p. Indeed, suppose $\lambda$ is the Cantor measure on $C$. Let $\mu_{1}, \mu_{2}, \mu_{3}, \mu_{4}$ and $\rho$ be the measures obtained respectively as images of $\lambda$ under the (bicontinuous) maps $x \rightarrow a_{1}(x)$, $x \rightarrow a_{2}(x), \quad x \rightarrow a_{1}(x)-b_{0}(x)+a_{0}(x), \quad x \rightarrow a_{2}(x)-b_{0}(x)+a_{0}(x)$, and $x \rightarrow$ $a_{1}(x)+a_{2}(x)-b_{0}(x)+a_{0}(x)$. Then $\mu_{1}, \cdots, \mu_{4}$ are mutually singular measures in $M_{c}(Q)$ and we have

$$
\rho \ll \mu_{1} * \mu_{4} \text { and } \rho \ll \mu_{2} * \mu_{3} .
$$

But there exists a linear functional $L$ of norm 1 on $M_{c}(Q)$ such that $L(\sigma)=0$ for $\sigma \ll \mu_{1}$ and $L(\sigma)=\sigma(G)$ for $\sigma \ll \mu_{j}, j=2,3,4$. If $M_{c}(Q)$ has the m.e.p. then there exists a multiplicative linear functional $M$ on $M(G)$ that extends $L$. By the results of Šrelder [6], $M$ is represented by a generalized character, i.e., a function $\chi$ on $M(G) \times G$ such that for each $\sigma \in M(G), \chi(\sigma,$.$) is Borel measurable and M(\sigma)=\int \chi(\sigma, x) \sigma(d x)$. Combining a basic property of generalized characters ([6], equation (35)), namely

$$
\chi(\sigma * \psi, s+t)=\chi(\sigma, s) \chi(\psi, t) \text { a.e. }(\sigma \times \psi),
$$

with the fact that

$$
\int \alpha(z) \sigma * \psi(d z)=\iint \alpha(s+t) \sigma(d s) \psi(d t),
$$

we see that $\chi\left(\mu_{1} * \mu_{4}, t\right)=0$ a.e. $\left(\mu_{1} * \mu_{4}\right)$ and $\chi\left(\mu_{2} * \mu_{3}, t\right)=1$ a.e. $\left(\mu_{2} * \mu_{3}\right)$. Since $\chi(\rho, t)=\chi\left(\mu_{1} * \mu_{4}, t\right)=\chi\left(\mu_{2} * \mu_{3}, t\right)$ a.e. $(\rho)$ ([6], Definition 1), we have a contradiction.

4. The disc algebra. Suppose $B$ is an arbitrary Banach algebra with unit and maximal ideal space $X$. Let $\mathfrak{F}$ be a family of linearly independent elements of $B$ of norm 1 . Denote by $[\mathfrak{F}]\left([\mathfrak{F}]^{-}\right)$the linear span (closed linear span) of $\widetilde{F}$. The following elementary proposition is useful in constructing subspaces of $B$ with the m.e.p.

Proposition. If for any $\left\{f_{1}, \cdots, f_{n}\right\} \subset \mathfrak{F}$ and any points $z_{1}, \cdots, z_{n}$ in $\vec{D}=\{z:|z| \leqq 1\}$, there exists an $M \in X$ with $M\left(f_{j}\right)=z_{j} j=1, \cdots, n$, then [₹] - has the m.e.p.

Proof. If $L$ is a linear functional on $\left[\mathfrak{F}^{-}\right.$with $\|L\| \leqq 1$, the hypothesis states that the closed sets

$$
\{M \in X: M(f)=L(f)\}
$$

for $f \in \mathfrak{F}$ have the finite intersection property. The conclusion is then 
immediate from the compactness of $X$.

We denote by $A(D)$ the algebra of functions continuous on $\bar{D}$ and analytic in $D$.

THEOREM 4. There exist infinite dimensional subspaces of $A(D)$ with the m.e.p.

Proof. By the transformation $x \rightarrow e^{2 \pi i x}$ of the unit interval we obtain a Cantor set on the boundary of $D$. By abuse of language we keep all the notation of Section 2, and regard $C, E_{x}, h_{x}$ as the objects induced by this transformation.

Let $\bar{D}^{\omega}$ be the product of $\bar{D}$ with itself a countable number of times. Since this is a compact metric space, there is a continuous map $\varphi$ of $C$ onto $\bar{D}^{\omega}$.

For $\sigma \in \bar{D}^{\omega}$, let $E_{\sigma}=\cup\left\{E_{x}: \varphi(x)=\sigma\right\}$. Each $E_{\sigma}$ is a perfect subset of $C$. In fact $E_{\sigma}=H\left[\varphi^{-1}(\{\sigma\}) \times E_{0}\right]$. Also the $E_{\sigma}$ are pairwise disjoint and $C=\cup\left\{E_{\sigma}: \sigma \in \bar{D}^{\omega}\right\}$.

Define a sequence $\left\{f_{n}\right\}$ of functions on $C$ as follows. For $\zeta \in C$, choose $\sigma$ so that $\zeta \in E_{\sigma}$, and set $f_{n}(\zeta)=\sigma(n)$. Then each $f_{n}$ is continuous on $C$. For suppose $\zeta_{j} \rightarrow \zeta_{0}, \zeta_{j} \in C$. Then each $\zeta_{j} \in E_{x_{j}}$ for some $x_{j} \in C$, and $\varphi\left(x_{j}\right)=\sigma_{j} \in \bar{D}^{\omega}$, and $\zeta_{0} \in E_{x_{0}}$ with $\varphi\left(x_{0}\right)=\sigma_{0}$. By 2.3, $x_{j} \rightarrow x_{0}$. Since $\varphi$ is continuous $\sigma_{j} \rightarrow \sigma_{0}$. Thus

$$
f_{n}\left(\zeta_{j}\right)=\sigma_{j}(n) \rightarrow \sigma_{0}(n)=f_{n}\left(\zeta_{0}\right) .
$$

Also clearly $\left\|f_{n}\right\|=1$ for each $n$.

By Rudin's theorem on extension of continuous functions to analytic functions [3, p. 81], for each $n$ there is a function $F_{n} \in A$ which agrees with $f_{n}$ on $C$ and $\left|F_{n}(z)\right| \leqq 1$ for $z \in \bar{D}$. If we let

$$
\mathfrak{\mho}=\left\{F_{1}, F_{2}, \cdots\right\} \text {, }
$$

it is clear that the hypotheses of the proposition are satisfied and thus $[\mathfrak{F}]^{-}$has the m.e.p., and the theorem is proved.

The following properties of the functions $F_{n}$ in the above example are noteworthy:

(i) $\left\|F_{n}+F_{m}\right\|=2$ for all $n, m$

(ii) $F_{n}^{-1}(\{w\})$ is uncountable for every $w \in \bar{D}$ and every $n$.

(iii) $F_{m}\left[F_{n}^{-1}(\{w\})\right]=\bar{D}$ for every $w \in \bar{D}$ and every pair $n, m$ with $n \neq m$.

(iv) If $f$ and $g$ are any two linearly independent elements of $[\mathfrak{F}]^{-}$, then for some $r, 0<r \leqq 1$,

$$
g\left[f^{-1}(\{0\})\right]=\{z:|z| \leqq r\} .
$$


Subspaces with the m.e.p. may be constructed in this manner in any algebra $B$ whose maximal ideal space contains a set $K$ homeomorphic to $C$ with the following property. If $f \in C(K)$ there is an $x \in B$ such that $\|x\|=\|f\|_{\infty}$ and the Gelfand transform of $x$ agrees with $f$ on $K$. For example, the algebra of continuous functions on the Stone-Cech compactification of the reals has this property.

\section{REFERENCES}

1. E. Hewitt, Measure algebras on locally compact groups: a case history in functional analysis, Studia Math. (ser. spec.) Z.I, (1963), 41-52.

2. E. Hewitt and S. Kakutani, A class of multiplicative linear functionals on the measure algebra of a locally compact abelian group, Illinois J. Math. 4 (1960), 553-574. 3. K. Hoffman, Banach Spaces of Analytic Functions, Prentice-Hall, Englewood Cliffs, 1962.

4. R. Phelps, A problem of Hewitt on restrictions of multiplicative linear functionals, Studia Math. 25 (1964), 1-3.

5. W. Rudin, Fourier Analysis on Groups, Interscience, New York, 1962.

6. Yu. A. Šreĩder, The structure of maximal ideals in rings of measures with convolution, Mat. Sbornik (69) 27 (1950), 297-318: AMS Translation No. 81, (1953).

UNIVERSITY OF BRITISH COLUMBIA 



\title{
PACIFIC JOURNAL OF MATHEMATICS
}

\author{
EDITORS
}

\section{H. SAMELSON}

Stanford University

Stanford, California

R. M. Blumenthal

University of Washington

Seattle, Washington 98105

\section{*J. DugundJI}

University of Southern California Los Angeles, California 90007

\section{RichaRd ARENS}

University of California

Los Angeles, California 90024

\section{ASSOCIATE EDITORS}
E. F. BECKENBACH
B. H. NEUManN
F. WOLF
K. YOSIDA

\section{SUPPORTING INSTITUTIONS}

\author{
UNIVERSITY OF BRITISH COLUMBIA \\ CALIFORNIA INSTITUTE OF TECHNOLOGY \\ UNIVERSITY OF CALIFORNIA \\ MONTANA STATE UNIVERSITY \\ UNIVERSITY OF NEVADA \\ NEW MEXICO STATE UNIVERSITY \\ OREGON STATE UNIVERSITY \\ UNIVERSITY OF OREGON \\ OSAKA UNIVERSITY \\ UNIVERSITY OF SOUTHERN CALIFORNIA
}

\author{
STANFORD UNIVERSITY \\ UNIVERSITY OF TOKYO \\ UNIVERSITY OF UTAH \\ WASHINGTON STATE UNIVERSITY \\ UNIVERSITY OF WASHINGTON \\ AMERICAN MATHEMATICAL SOCIETY \\ CHEVRON RESEARCH CORPORATION \\ TRW SYSTEMS \\ NAVAL ORDNANCE TEST STATION
}

\footnotetext{
Mathematical papers intended for publication in the Pacific Journal of Mathematics should be typewritten (double spaced). The first paragraph or two must be capable of being used separately as a synopsis of the entire paper. It should not contain references to the bibliography. Manuscripts may be sent to any one of the four editors. All other communications to the editors should be addressed to the managing editor, Richard Arens at the University of California, Los Angeles, California 90024 .

50 reprints per author of each article are furnished free of charge; additional copies may be obtained at cost in multiples of 50 .
}

The Pacific Journal of Mathematics is published monthly. Effective with Volume 16 the price per volume (3 numbers) is $\$ 8.00$; single issues, $\$ 3.00$. Special price for current issues to individual faculty members of supporting institutions and to individual members of the American Mathematical Society: $\$ 4.00$ per volume; single issues $\$ 1.50$. Back numbers are available.

Subscriptions, orders for back numbers, and changes of address should be sent to Pacific Journal of Mathematics, 103 Highland Boulevard, Berkeley 8, California.

Printed at Kokusai Bunken Insatsusha (International Academic Printing Co., Ltd.), No. 6, 2-chome, Fujimi-cho, Chiyoda-ku, Tokyo, Japan.

PUBLISHED BY PACIFIC JOURNAL OF MATHEMATICS, A NON-PROFIT CORPORATION

The Supporting Institutions listed above contribute to the cost of publication of this Journal, but they are not owners or publishers and have no responsibility for its content or policies.

* Paul A. White, Acting Editor until J. Dugundji returns. 


\section{Pacific Journal of Mathematics}

\section{Vol. 17, No. $3 \quad$ March, 1966}

Tsuyoshi Andô, Contractive projections in $L_{p}$ spaces ............... 391

Robert F. Brown, On a homotopy converse to the Lefschetz fixed point theorem ............................................... 407

Richard Albert Cleveland and Sandra Cleveland, On the multiplicative extension property................................ 413

Harold H. Johnson, An algebraic approach to exterior differential systems..................................... 423

Alan Cecil Lazer, The behavior of solutions of the differential equation

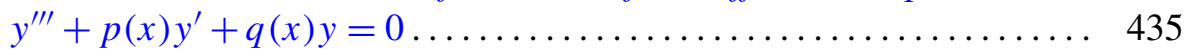

Judy Parr, Cohomology of cyclic groups of prime square order ......... 467

Donald Steven Passman, Groups whose irreducible representations have

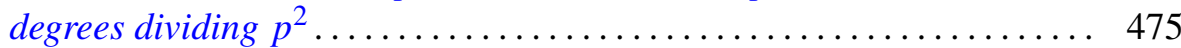

Ralph Tyrrell Rockafellar, Characterization of the subdifferentials of convex functions ........................................... 497

Donald Erik Sarason, Invariant subspaces and unstarred operator algebras...................................... 511

Donald Erik Sarason, Weak-star generators of $H^{\infty} \ldots \ldots \ldots \ldots \ldots \ldots . \ldots . \ldots$

Boris M. Schein, Homomorphisms and subdirect decompositions of semi-groups

Daniel Francis Shea, Jr., Functions analytic in a finite disk and having asymptotically prescribed characteristic

Zvi Ziegler, Generalized convexity cones 